\title{
Herbicide injury induces DNA methylome alterations in Arabidopsis
}

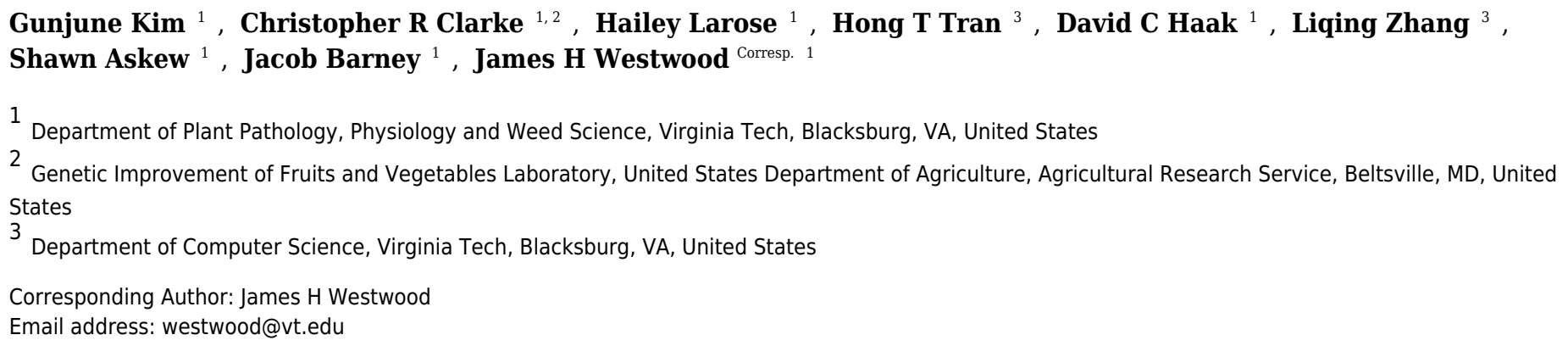

The emergence of herbicide-resistant weeds is a major threat facing modern agriculture. Over 470 weedy plant populations have developed resistance to herbicides. Traditional evolutionary mechanisms are not always sufficient to explain the rapidity with which certain weed populations adapt in response to herbicide exposure. Stress-induced epigenetic changes, such as alterations in DNA methylation, are potential additional adaptive mechanisms for herbicide resistance. We performed methylC sequencing of Arabidopsis thaliana leaves that developed after either mock treatment or two different sub-lethal doses of the herbicide glyphosate, the most used herbicide in the history of agriculture. The herbicide injury resulted in 9205 differentially methylated regions (DMRs) across the genome. 5914 of these DMRs were induced in a dose-dependent manner, wherein the methylation levels were positively correlated to the severity of the herbicide injury, suggesting that plants can modulate the magnitude of methylation changes based on the severity of the stress. Of the 3680 genes associated with glyphosate-induced DMRs, only $7 \%$ were also implicated in methylation changes following biotic or salinity stress. These results demonstrate that plants respond to herbicide stress through changes in methylation patterns that are, in general, dose-sensitive and, at least partially, stressspecific. 
1 Title: Herbicide injury induces DNA methylome alterations in Arabidopsis

2 Authors: Gunjune Kim ${ }^{a *}$ Christopher R. Clarke ${ }^{a b *}$, Hailey Larose ${ }^{\mathrm{a}}$, Hong Tran ${ }^{\mathrm{c}}$, David C.

3 Haak $^{\mathrm{a}}$, Liqing Zhang ${ }^{\mathrm{c}}$, Shawn Askew ${ }^{\mathrm{a}}$, Jacob Barney ${ }^{\mathrm{a}}$, James H. Westwood ${ }^{\mathrm{a}}{ }^{\dagger}$

5 Affiliations:

6 a Department of Plant Pathology, Physiology, and Weed Science. Virginia Tech. Blacksburg, 7 VA, USA.

$8{ }^{\mathrm{b}}$ Current address: Genetic Improvement of Fruits and Vegetables Laboratory, United States

9 Department of Agriculture, Agricultural Research Service; Beltsville Agricultural Research

10 Center

$11{ }^{\mathrm{c}}$ Department of Computer Science. Virginia Tech. Blacksburg, VA, USA.

12

*Equal contribution

14 †Correspondence to: James Westwood, westwood@vt.edu

\section{Abstract}

The emergence of herbicide-resistant weeds is a major threat facing modern agriculture. Over mechanisms are not always sufficient to explain the rapidity with which certain weed populations adapt in response to herbicide exposure. Stress-induced epigenetic changes, such as alterations in DNA methylation, are potential additional adaptive mechanisms for herbicide resistance. We performed methylC sequencing of Arabidopsis thaliana leaves that developed after either mock treatment or two different sub-lethal doses of the herbicide glyphosate, the most used herbicide in the history of agriculture. The herbicide injury resulted in 9205 differentially methylated regions (DMRs) across the genome. 5914 of these DMRs were induced in a dose-dependent manner, wherein the methylation levels were positively correlated to the severity of the herbicide injury, suggesting that plants can modulate the magnitude of methylation changes based on the severity of the stress. Of the 3680 genes associated with glyphosate-induced DMRs, only 7\% were also implicated in methylation changes following biotic or salinity stress. These results demonstrate that plants respond to herbicide stress through changes in methylation patterns that are, in general, dose-sensitive and, at least partially, stressspecific. 


\section{Introduction}

The development of herbicide-resistant weed populations is a major crisis facing modern agriculture (Bonny 2016). Herbicide resistance has evolved in populations of at least 470 different weeds, including 35 species that have developed resistance to the herbicide glyphosate (Heap 2016), the most widely used herbicide in the history of agriculture (Duke \& Powles 2008). Mechanisms of herbicide resistance are easily explained in some cases, as when a mutation occurs in the protein target of the herbicide that reduces herbicide binding (González-Torralva et al. 2012) or even the copy number of herbicide target genes (Gaines et al. 2010). Other cases, termed non-target site resistance, are poorly understood, but have been attributed to quantitative accumulation of minor resistance alleles selected from standing genetic variation at multiple gene loci (Busi et al. 2013; Délye 2013). Non-target site resistance may involve various mechanisms that affect herbicide metabolism or translocation (González-Torralva et al. 2012). Resistance to glyphosate is interesting in that it often appears to involve non-target site mechanisms, which can emerge and dominate a population after as few as three generations of sub-lethal exposure to the herbicide (Busi \& Powles 2009), and can spread through a population faster than predicted by gene flow or propagule dispersal (Escorial et al. 2011; Espeby et al. 2011; Okada et al. 2013). Given that herbicides induce a strong abiotic stress, it is likely that weeds respond by activating stress signaling networks that reprogram gene expression (Busi et al. 2013), and we hypothesize that this involves epigenetic regulation of gene function that could contribute to herbicide resistance as implicated in weed adaptation to other stresses (e.g. Verhoeven et al. 2010) and in the response of rice to the pesticide atrazine (Lu et al. 2016).

A prominent mechanism of epigenetic regulation of gene expression is through cytosine methylation. Methylated cytosines (mCs) occur in three different sequence contexts in plants: $\mathrm{CG}$ methylation, which is the predominant form of methylation in gene rich regions, and $\mathrm{CHH}$ or $\mathrm{CHG}(\mathrm{H}=\mathrm{A}, \mathrm{T}$, or $\mathrm{C})$ methylation predominantly in transposable elements and repetitive sequences (Cokus et al. 2008). CHH and CHG mCs are much more common in plants than animals (Cokus et al. 2008). Multiple RNA-dependent methylation pathways control de novo methylation in the mustard weed Arabidopsis thaliana (Matzke \& Mosher 2014) with methylation at the different sequence contexts altered and maintained through both overlapping and sequence-context-specific mechanisms. Mutations in the genes of these pathways lead to aberrations in the methylome (Stroud et al. 2013). Additionally, wildtype $A$. thaliana plants accumulate epimutations (i.e. changes in the methylome) over generations of greenhouse propagation (Becker et al. 2011; Schmitz et al. 2011). Stress exposure can lead to substantial methylome reprogramming as observed in A. thaliana following pathogen attack or salicylic acid treatment (Dowen et al. 2012), phosphate starvation (Secco et al. 2015; Yong-Villalobos et al. 2015), and salinity stress (Jiang et al. 2014; Wibowo et al. 2016). The majority of methylation changes following these stresses were considered transient - not transgenerationally stable - but a subset of stress-responsive methylations is stably fixed in populations over several subsequent generations of exposure to stress (Jiang et al. 2014). Thus, transgenerational epigenetic changes 
in gene expression could lead to enhanced and adaptive stress tolerance. However, the ability of epigenetic changes to be transgenerationally stable and affect the adaptation of plants in their environment remains contentious (Hagmann et al. 2015; Kawakatsu et al. 2016).

Because the specificity of methylome reprogramming following stress may appear inexact (e.g., compare Secco et al. 2015 to Yong-Villalobos et al. 2015) and the effect of alterations in methylation levels on gene expression may seem inconsistent (e.g. Karan et al. 2012; Kawakatsu et al. 2016; Li et al. 2012), we tested whether there was a dosage effect by glyphosate stress on specific alterations of the plant methylome. Glyphosate has been previously shown to alter DNA methylation in wheat (Nardemir et al. 2015) using relatively low resolution techniques.

Additionally, the herbicide atrazine was recently shown to induce global changes in methylation in rice (Lu et al. 2016). We employed bisulfite sequencing to determine the effect of glyphosate on the Arabidopsis methylome at single base pair resolution to identify specific genetic loci that have altered methylation patterns following glyphosate exposure.

\section{Materials and Methods}

$\underline{\text { Plant growth conditions and herbicide treatment }}$

Seeds of $A$. thaliana ecotype Columbia were sown in Sunshine Number 1 media and stratified for three days in the dark at $4^{\circ} \mathrm{C}$. The flats were then transferred to a Conviron growth chamber with 12-hour light cycle and light intensity of $90 \mu \mathrm{mol} \mathrm{m} \mathrm{m}^{-2} \mathrm{~s}^{-1}$ and allowed to grow to a fully developed rosette, pre-floral shoot stage. Blocks of 4 plants were then randomly assigned treatment of glyphosate at $0 \%, 5 \%, 10 \%$, or $15 \%$ of the label rate $(0.9 \mathrm{~kg}$ acid equivalency (ae) $\mathrm{ha}^{-1}$ of RoundUp Pro Concentrate). Glyphosate-treated plants were sprayed at $187 \mathrm{~L} \mathrm{ha}^{-1}$ in a spray booth. Following glyphosate treatment, plants were transferred to a growth shelf with a 12hour light cycle and light intensity of $90 \mu \mathrm{mol} \mathrm{m} \mathrm{m}^{-2} \mathrm{~s}^{-1}$ and grown until fully-developed siliques were formed (approximately 2 weeks for the $0 \%$ and 5\% glyphosate-treated plants and 8 weeks for the $10 \%$ glyphosate-treated plants). Thus, tissues were harvested at equivalent developmental stages, despite slower growth rate of glyphosate-treated plants.

\section{$\underline{\text { Genomic DNA isolation and MethylC sequencing library preparation }}$}

Genomic DNA was isolated from 2-3 cauline leaves formed following glyphosate exposure from individual plants in quadruplicate for each of the three treatment levels $(0 \%, 5 \%$ and $10 \%)$ using the Biosprint-15 plant DNA extraction kit (Qiagen, Hilden, Germany). The 12 samples were sent to Genomics Research Laboratory at Biocomplexity Institute of Virginia Tech (Blacksburg, VA, USA) for library preparation and bisulfite sequencing. $100 \mathrm{ng}$ of intact DNA was bisulfite converted using EZ DNA Methylation-Gold Kit (\#D5005, Zymo Research), following the manufacturers protocol, except eluting into $9 \mathrm{ul}$. The entire amount of the purified bisulfitetreated DNA was converted to Illumina DNA libraries using EpiGnome Methyl-Seq kit (Epicentre). Six samples each were individually barcoded, quantitated by qPCR and pooled to sequence on the entire Illumina HiSeq Rapid Run flowcell. Libraries were clustered on-board at 
111 a concentration of $8.5 \mathrm{pM}$ with $3 \%$ phiX, onto a flow cell using Illumina's HiSeq Rapid Paired

112 End Cluster Kit V2 (PE-402-4002), and sequenced 2x 101 cycles using HiSeq Rapid SBS Kit

113 (200-cycles) (FC-402-4021).

114 MethylC sequencing data analysis

115 The sequencing reads were subject to pre-processing quality control using FastQC to eliminate

116 adapter sequences and barcodes using Trimmomatic (http:/www.usadellab.org/cms/) and FastX

117 Tookit (http://hannonlab.cshl.edu/fastx toolkit/). Low quality reads (quality score $\mathrm{Q}<30$ ) were

118 discarded and only reads passing the quality check were mapped to Col-0 Arabidopsis thaliana

119 (TAIR 10) reference genome using Bismark aligner (v 0.14.5) under default parameters (-n 1 -1

120 50) (Krueger \& Andrews 2011). Cytosine methylation information was extracted from aligned

121 reads using Bismark methylation extractor and methylation calls for $\mathrm{CG}, \mathrm{CHH}$, and $\mathrm{CHG}$

122 contexts were generated. The conversion efficiency of bisulfite treatment (methylation status of

123 cytosine) was estimated from reads mapped to the chloroplast genome, which is expected to be

124 unmethylated.

125

126

127

128

129

130

131

132

133

134

135

136

137

138

139

140

141

142

143

144

145

146

\section{$\underline{\text { Calling DMRs }}$}

\section{Methylkit/eDMR}

DMRs (differentially methylated regions) between treated and control plants were identified using the R (v3.0.3) package methylkit/eDMR (Li et al. 2013). Methylkit allows parameter adjustment to identify DmCs based on q-value, percent methylation difference, and types of methylation (hyper or hypo) using statistical tests such as logistic regression and Fisher's exact test. Pairwise Pearson's correlation coefficient and Hierarchical clustering (Ward's method, correlation distance metric) were calculated based on percent methylation values for all 12 samples. Differential methylation between treated (5\% and 10\%) and control groups were determined using Fisher's exact test with a minimum $25 \%$ difference in methylation ratio between groups and q-value $<0.01$. DMRs were constructed using weighted optimization algorithm eDMR.

bsseq

Differential methylation between glyphosate-treated and control libraries was also determined in $\mathrm{R}$ using bsseq package (bioconductor). The coverage (.cov) files generated by Bismark were used to run methylation smoothing which generates per- $\mathrm{CG}, \mathrm{CHH}$ and $\mathrm{CHG}$ methylation values based on at least one biological replicate for control and treated samples using mean t-statistics. DMRs were filtered by areaStat which was weighted by the number of methylation sites for each context (Hansen et al. 2012). We identified the overlapping DMR regions for each context between percent of glyphosate exposure using the intersectBed function within the bedtools suite with default parameters (Quinlan \& Hall 2010). The stringency of the parameters to use when calling DMRs was optimized through analyzing the effect of increasing the number of analyzed 
147 replicates on the number of identified DMRs by calling DMRs using all possible combinations of

1482 replicates, 3 replicates and 4 replicates from our methylC-seq data. For the less stringent

149 parameters (See Figure S1 legend) relying on two replicates likely leads to many false positives

150 because of the sharp decline in the number of DMRs when increasing the number of replicates

151 (Figure S1). When using the more stringent parameters, the overall number of DMR calls is

152 lower and increasing replicates has no effect on raw number of called DMRs suggesting fewer

153 false positives. We therefore used the DMRs called using the more stringent parameters for

154 downstream analyses.

155 Gene Ontology Enrichment Analysis

156 Annotations from the TAIR10 database were assigned to DMRs using a custom Perl script

157 (script provided in supplementary materials) in which genomic features were associated with

158 DMRs that overlapped within $2 \mathrm{~Kb}$ in either direction. Arabidopsis thaliana ID lists from

159 associated DMRs were processed and analyzed in VirtualPlant 1.3 software

160 (http://virtualplant.bio.nyu.edu/cgi-bin/vpweb/) using the BioMaps module $(\mathrm{P} \leq 0.05)$ to find

161 significantly over-represented gene ontology (GO) categories. In order to select significant

162 functional categories, we set a cutoff point in a normalized frequency (relative frequency of input

163 gene list/relative frequency of reference) that was greater than 1.5-fold.

164 Classification of dose-dependent response of DMRs identified in both 5\% and 10\% datasets

165 Statistical hypothesis tests using the Student's t-test with 5\% significance levels were conducted

166 to determine if the mean difference in 5\% glyphosate vs. control group $\left(\Delta_{5}\right)$ is significantly

167 different from the mean difference in $10 \%$ glyphosate vs. control group $\left(\Delta_{10}\right)$ for each DMR. We

168 then fitted one nonlinear curve for sites in which $\Delta_{5}$ is significantly greater than $\Delta_{10}$ and all

169 DMRs below the fitted blue curve in hypermethylation case and above the fitted blue curve in

170 hypomethylation case were classified as inverse dose-dependent (see Figure S2). Another

171 nonlinear curve for sites in which $\Delta_{5}$ is significantly less than $\Delta_{10}$ was then fitted. All DMRs

172 above the fitted green curve in hypermethylation case and below the fitted green curve in

173 hypomethylation case were classified as positive dose-dependent. Nonlinear curves are

174 appropriate for fitting the data based on both Akaike information criterion (AIC) and Bayesian

175 information criterion (BIC). The nonlinear curve fitting AIC and BIC were smaller compared to

176 linear fitting.

177 Statistical comparison of overlapping DMR-associated genes across different stresses

178 (glyphosate, phosphate starvation, biotic)

179 The number of DMR-associated genes following glyphosate treatment, phosphate starvation and

180 biotic stress are 3680, 712 and 884, respectively (Dataset S7). Only 13 genes were identified as

181 being differentially methylated following all these stresses (Dataset S7). We considered the

182 possibility that the lack of shared DMR-associated genes among all three stresses is evidence for

183 DMRs being randomly distributed across the genome as opposed to selective in response to 
184

185

186

187

188

189

190

191

192

193

194

195

196

197

198

199

200

201

202

203

204

205

206

207

208

209

210

211

212

213

214

215

216

217

218

219

220

221

stress. The total number of genes in the TAIR10 annotation of the $A$. thaliana genome is 33,602. We included the total number of $A$. thaliana genes as the baseline rather than previously published lists of methylated $A$. thaliana genes because a majority of our genic DMRs (73\%) were identified in genes previously characterized as unmethlyated. The glyphosate-induced DMR-associated genes account for $11 \%$ of all possible genes. If these 3680 genes are randomly distributed across the genome, and the 884 biotic stress DMR-associated genes are likewise random, then the expected number of overlapping genes can be calculated by $0.11 * 884=97$. Using the same formula for the phosphate starvation DMR-associated genes the expected number of overlaps with the glyphosate DMR-associated genes is 78 if the DMRs are randomly distributed. Under the same assumptions the expected number of DMR-associated genes linked to all three stress responses is 2 [(97/33602)*712], much smaller than the observed 13 shared genes. In fact, Chi-square test of independence of the three stresses is highly significant $\left(\chi^{2}=\right.$ 179.39, $d f=4$, $\mathrm{p}$-value $=1.01 \mathrm{e}-37)$, suggesting that the three stresses indeed induce common epigenetic changes in A. thaliana. Moreover, all pairwise comparison test of independence shows statistical significance (glyphosate and phosphate: $\chi^{2}=62.21, d f=1, \mathrm{p}$-value $=3.088 \mathrm{e}-15$; glyphosate and biotic: $\chi^{2}=7.557, d f=1, \mathrm{p}$-value $=0.005977$; phosphate and biotic: $\chi^{2}=100.08$,

$d f=1$, p-value $=1.463 \mathrm{e}-23$ ). Taken together, the comparison result shows that DMR-associated genes are not randomly distributed in the Arabidopsis genome and DMR-associated genes in response to one stress are not mutually independent of those involved in response to another stress.

\section{Results and Discussion}

To determine whether glyphosate injury induces changes in plant methylation, we treated $A$. thaliana with low concentration glyphosate sprays representative of real-world doses a weed on the margin of a treated field could receive. Because a plant must survive herbicide injury and produce viable seed to be a founder of an herbicide resistant population, we first identified the appropriate sub-lethal doses for glyphosate treatment of $A$. thaliana. Four-week-old $A$. thaliana rosettes were exposed to $0,5,10$, or $15 \%$ of a typical field rate of $0.9 \mathrm{~kg}$ acid equivalency (ae) ha $^{-1}$ glyphosate, with the 5 and $10 \%$ rates causing visible herbicide injury, but allowing for plant survival and reproduction (Figure 1a). gDNA was collected from newly formed cauline leaves at silique maturation of four individuals from each treatment (Figure 1b-d). MethylC-seq of 12 total libraries (four replicates from each treatment) produced a total of 530,042,668 aligned reads resulting in genome coverages from $48 \mathrm{x}-76 \mathrm{x}$ for each replicate (Table S1).

The total amount of methylated cytosines ( $\mathrm{mCs}$ ) ranged from 95-143 million for all replicates (Table S1) and were the most abundant in the CG sequence context (Figure 2a), which is consistent with previous analyses of the $A$. thaliana methylome (Cokus et al. 2008). Only an average of $0.2 \% \mathrm{mCs}$ were identified on the chloroplast genome, which is not methylated in $A$. thaliana (Cokus et al. 2008), demonstrating the fidelity of the methylC-seq protocol and data filtering. mCs were distributed across all five nuclear chromosomes. Additionally, a small 
222 number of $\mathrm{mCs}$ were identified in the mitochondrial genome though these $\mathrm{mCs}$ are likely false

223

224

225

226

227

228

229

230

231

232

233

234

235

236

237

238

239

240

241

242

243

244

245

246

247

248

249

250

251

252

253

254

255

256

257

258

259

260

positives because of the insertion of a region of the mitochondrial genome on chromosome 2 of A. thaliana eco. Columbia (Lin et al. 1999). Neither the abundance of mCs nor the relative frequency of $\mathrm{mCs}$ in the three different analyzed sequence contexts (CG, $\mathrm{CHG}, \mathrm{CHH}$ ) significantly changed due to herbicide injury (Figure 2a). Clustering the Pearson's correlation coefficient of the mCs did not result in treatment-specific monophyletic clades, demonstrating the relative similarity of the methylomes across all 12 individuals (Figure S3). Taken together, these results suggest that glyphosate-induced stress did not lead to changes in overall levels of methylation in any of the three sequence contexts.

Because glyphosate did not induce global shifts in methylation levels, differences in specific location and context of DNA methylation can be potentially classified as selective responses to herbicide injury. We used Methylkit (Akalin et al. 2012) to compare mCs in the 5\% or 10\% glyphosate treatments to the control ( $0 \%$ treatment) to identify differentially methylated cytosines (DmCs). This approach enumerated 17,017 DmCs following 5\% glyphosate treatment and 23,341 DmCs following 10\% glyphosate treatment after normalizing all four replicates and applying a cutoff to only consider the top 25\% highest confidence DmCs (Dataset S1). Interestingly, $10 \%$ glyphosate treatment led to substantially more DmCs than 5\% glyphosate treatment in the two most abundant sequence contexts CG and CHG (Figure 2b-d), suggesting that the degree of herbicide injury is correlated with the context and magnitude of methylation changes. However, it is important to note that the $10 \%$ glyphosate-treated plants required an additional 6 weeks to reach silique maturity. Therefore, a subset of the methylation differences could be due to the increased growing time and not the herbicide treatment.

Differentially methylated regions (DMRs) are more strongly associated with regulatory changes in gene expression than DmCs. We called DMRs induced by both 5\% and 10\% glyphosate treatment using two independent approaches: a bimodal DmC distribution modeling approach using eDMR (Li et al. 2013), and a curve smoothing approach of DmCs using bsseq (Hansen et al. 2012). All DMRs were defined as CG, CHH, or CHG based on the predominance of the sequence context of DmCs comprising the DMR. The eDMR algorithm identified 1949 hypomethylated and 1229 hypermethylated non-redundant DMRs following 5\% and 10\% glyphosate treatments (Dataset S2). Greater than 95\% of the DMRs called by eDMR were defined by the CG sequence context (Figure S4). Using the bsseq algorithm, 4053 hypomethylated DMRs and 5082 hypermethylated DMRs were identified following glyphosate herbicide injury across all three sequence contexts (Dataset S3). We excluded 70 DMRs identified in both the $5 \%$ and $10 \%$ treatment groups but conflicting in directional change of methylation (i.e. hypo in one treatment and hyper in the other treatment) and therefore unlikely to represent biologically relevant DMRs. In contrast to the eDMR data, only $78 \%$ and $51 \%$ of hypomethylated and hypermethylated bsseq DMRs, respectively, were categorized as CG (Figure 3a). The lack of congruence between the bsseq and eDMR datasets suggests that the choice of algorithm remains a primary driver for the specificity of DMRs identified as previously 
261

262

263

264

265

266

267

268

269

270

271

272

273

274

275

276

277

278

279

280

281

282

283

284

285

286

287

288

289

290

291

292

293

294

295

296

297

298

suggested (Yu and Sun 2016). We consider the 545 DMRs identified via both eDMR and bsseq as our highest confidence DMRs (Dataset S3). We focused on the bsseq DMRs for subsequent analyses because of the relative abundance of non-CG DMRs in this dataset and the observation that non-CG DMRs are more frequent in plants than they are in animals.

We identified 1964 DMRs (counting both hypo- and hyper-methylated DMRS) unique to the 5\% glyphosate-treated samples, and 5614 DMRs unique to the 10\% samples (Figure 3a), which is consistent with the trend observed for DmCs in which the severity of herbicide injury correlates with the magnitude of methylome shifts. The 5614 DMRs present only in the $10 \%$ glyphosatetreated samples were tentatively classified as positive dose-dependent DMRs (i.e., the magnitude of changes in methylation levels are positively correlated with severity of herbicide injury) and the 1964 DMRs unique to the 5\% glyphosate-treated samples as inverse dose-dependent DMRs (i.e., more severe herbicide injury correlated with decreases in the magnitude of changes in methylation levels). The 1557 DMRs present in both the 5\% and 10\% treated samples were further analyzed for dose-dependency resulting in 224, 300, and 209 DMRs classified with 95\% confidence as dose-independent, positive dose-dependent, and inverse dose-dependent respectively, across both hypomethylated and hypermethylated DMRs (Figure 3b, Dataset S4, Figure S2, see Methods). We therefore conclude that for nearly two-thirds (5914 out of 9205) of DMRs that are positive dose-dependent, the magnitude of changes in methylation is dependent on the severity of the stress. We propose that these positive dose-dependent DMRs are the best candidates for identifying biologically relevant genomic loci that are modified in response to glyphosate stress. However, it is important to note that the $10 \%$ glyphosate-treated plants were harvested several weeks after the $5 \%$ glyphosate-treated plants in order to compensate for the developmental delay induced in the $10 \%$ glyphosate-treated plants (see Methods). It is possible that a number of the identified differential DMRs between the two treatment levels are due to maintenance of these DMRs being uncoupled from development and instead linked to the age of the plants.

We tentatively hypothesize for the smaller number of the inverse dose-dependent DMRs that the severity of herbicide injury following $10 \%$ glyphosate treatment prevented the plant from responding to the stress through specific alterations in DNA methylation. Interestingly, the $\mathrm{CHH}$ context had the most pronounced inverse dose-dependent trend, with more DmCs in the 5\% treatment group than the 10\% treatment group (Figure 2), in stark contrast to the $\mathrm{CG}$ and $\mathrm{CHG}$ contexts. While some molecular mechanisms that control methylation act on all three sequence contexts (e.g. DRM 1/2 (Cao \& Jacobsen 2002b)) other mechanisms have distinct effects across different sequence contexts with the most pronounced differences between asymmetrical and symmetrical Cs (Cao \& Jacobsen 2002a; Matzke \& Mosher 2014). CHH sites are asymmetrical (no $\mathrm{C}$ in the antisense strand) in contrast to $\mathrm{CG}$ and $\mathrm{CHG}$ sites. Additionally, $\mathrm{CHH}$ sites were previously identified as responding uniquely to biotic stress in contrast to $\mathrm{CG}$ and $\mathrm{CHG}$ sites (Dowen et al. 2012). However, it is impossible to conclude that glyphosate herbicide injury alters 
299

300

301

302

303

304

305

306

307

308

309

310

311

312

313

314

315

316

317

318

319

320

321

322

323

324

325

326

327

328

329

330

331

332

333

334

335

336

337

different molecular methylation mechanisms specifically until the effects of glyphosate on methylation patterns in known methylation mutant plant lines are empirically assessed.

To elucidate which genomic regions are responding to the glyphosate stress through changes in methylation we annotated all DMRs. A plurality of DMRs were identified in gene coding sequences and in the CG context (Figure 4a) as expected (Cokus et al. 2008). The DMRs categorized as $\mathrm{CHG}$ or $\mathrm{CHH}$ were predominantly associated with transposable elements, similar to previous studies in plants (Cokus et al. 2008; Li et al. 2012). In total, 1818 transposable elements or transposable element (TE) genes were hypermethylated and 936 were hypomethylated (Figure 4a, Dataset S3). Transposable element-associated DMRs occurred at varying frequency across superfamily groups in response to both the $5 \%$ and $10 \%$ glyphosate treatments (Figure S5, Dataset S5). Even though hypermethylated TEs are twice as common as hypomethylated TEs, the 936 hypomethylated TEs may contribute to genome destabilization due to increased transposon mobility associated with reduced transposon body methylation (Chan et al. 2005; Mirouze \& Vitte 2014). The number of hypomethylatedTEs is less than the number of activated TEs in the DNA methylation A. thaliana mutant ddm1 (Zemach et al. 2013), but still greater than the number of TEs activated in $A$. thaliana following treatment with DNA demethylating agent azacytidine based on expression data (Griffin et al. 2016). We therefore conclude that glyphosate induces hypomethylation of TEs on a scale comparable to other known treatments capable of destabilizing the $A$. thaliana genome through TE hypomethylation. The hypomethylated TEs are interesting to explore in terms of their potential for contributing to the phenomenon of gene amplification observed in several glyphosate resistant weeds (Gaines et al. 2010).

An advantage to studying plant DNA methylation changes in response to glyphosate is that the biochemical mechanism of action of this herbicide has been extensively characterized. Glyphosate stops the flow of carbon through the shikimate pathway by inhibiting activity of the enzyme 5-enolpyruvylshikimate 3-phosphate (EPSP) synthase (Amrhein et al. 1980). Two of the seven genes in the shikimate pathway of Arabidopsis (Tzin \& Galili 2010) were differentially methylated in the CG context in response to glyphosate stress: 3-deoxy-d-arabino-heptulosonate7-phosphate (DAHP) synthase (At4g33510), which encodes the first enzyme (and major regulatory step for the pathway), and both forms of shikimate kinase (SK1, At2g21940 and SK2, At4g39540) were all hypermethylated in the 10\%-glyphosate treated plants. Notably, all three of these genes were previously classified as unmethylated in A. thaliana (Niederhuth et al. 2016). EPSP synthase (At2g45300), which is also considered unmethylated in A. thaliana populations (Niederhuth et al. 2016), was not differentially methylated due to glyphosate injury (Dataset S3). Several biological processes and molecular functions were enriched in the Gene Ontology (GO) terms linked to the DMR-associated genes (genes that contain DMRs in their coding sequence) (Figure 4b-c, Dataset S6). The enrichment of genes associated with phosphate (including phosphorylation, phosphotransferase, protein kinase and pyrophosphatase) was striking. This could be related to glyphosate induction of microRNAs that regulate phosphate transport 
338

339

340

341

342

343

344

345

346

347

348

349

350

351

352

353

354

355

356

357

358

359

360

361

362

363

364

365

366

367

368

369

370

371

372

373

374

375

376

pathways observed in maize (Żywicki et al. 2015). The glyphosate molecule is recognized by phosphate transporters (Denis \& Delrot 1993) and some cases of glyphosate resistance have been attributed to alterations in herbicide transport and sequestration (Sammons \& Gaines 2014).

As expected, the majority of the enriched GO terms were associated with genic DMRs in the CG context (Figure S6, Dataset S6), and these included many of the terms related to phosphate. The $\mathrm{GO}$ terms enriched in the $\mathrm{CHG}$ and $\mathrm{CHH}$ contexts were distinct. In the $\mathrm{CHG}$ context, glyphosate herbicide injury was associated with alterations in methylation of cell wall-associated and hydrolase genes. Genes associated with signal reception and transduction were specifically enriched in this analysis for genic DMRs in the CHH context.

Formation and maintenance of DMRs is driven by changes in gene expression (Secco et al. 2015), and DMRs are sometimes associated with downstream changes in gene expression (Li et al. 2012) though often have no effect on gene expression (Kawakatsu et al. 2016). We therefore cannot conclude whether glyphosate is directly altering the methylation patterns of the genes associated with phosphate metabolism and the shikimate pathway or if glyphosate is altering transcription of associated genes that in turn modify the methylation states of the underlying genomic loci. Previous work showed that glyphosate caused only minor changes in immediate transcriptional activity in A. thaliana eco. Columbia (Das et al. 2010), but induced major effects on the transcriptome of $A$. thaliana eco. Lansberg erecta (Faus et al. 2015). Future work including paired transcriptome analyses are required to correlate methylation patterns with gene expression.

We next asked whether the DMRs induced by glyphosate treatment are indicative of a general stress response or potentially at least partially glyphosate specific. We compared the 3680 nonredundant DMR-associated genes in either the $5 \%$ or $10 \%$ treatment group to DMR-associated genes that were previously identified following salicylic acid (SA) treatment, which mimics biotic stress (Dowen et al. 2012), or phosphate starvation stress (Yong-Villalobos et al. 2015). Even considering the use of different approaches and cutoffs for calling DMRs, we hypothesized that the three stresses would induce overlapping methylome alterations as a result of the plants' general response to environmental stresses and would therefore share some DMR-associated genes. Only 13 genes were identified as being differentially methylated following all three stress treatments (Figure 4d, Dataset S7). These 13 DMR-associated genes are top candidates for genes that are epigenetically regulated as part of a general stress response. An additional 109 and 130 glyphosate-induced DMRs overlapped with DMRs associated with biotic stress and phosphate starvation stress, respectively. 3428 (93\%) of the glyphosate-induced-DMRassociated genes were unique to glyphosate exposure. While the number of overlapping DMRassociated genes among the three analyzed stresses seems small, they represent significantly more than would occur by random selection from all $A$. thaliana genes (see Methods). Moreover, Chi-square tests of independence of DMR-associated genes in three stresses show that stress responses are not independent of one another $\left(\chi^{2}=179.39, d f=4\right.$, p-value $\left.=1.01 \mathrm{e}-37\right)$, and there is indeed a higher than expected number of genes involved in response to all three 
377 stresses, indicating the existence of a common methylome reprogramming pathway in

378 Arabidopsis regardless of the stressors. Nevertheless, the majority of identified DMRs appear

379 specific to glyphosate injury.

\section{Conclusion}

381

382

383

384

385

386

387

388

389

390

391

392

393

394

395

396

397

398

399

400

401

402

403

404

405

406

407

408

409

410

411

412

This work identifies a large set of genes and other genomic regions epigenetically regulated in response to glyphosate herbicide injury. Determining the extent to which stresses induce patterned versus random epimutations is critical for understanding the role of DNA methylation in plant adaptation to stresses. We favor the hypothesis that the methylome changes are, at least partially, stress specific over the hypothesis that methylome alterations are universally random because: 1) A majority of the DMRs exhibit dose-sensitive response patterns; 2) all analyzed glyphosate-induced DMRs were identified in four independent biological replicates; and 3) DMRs were enriched in gene pathways known to be affected by glyphosate exposure. Identification of transgenerationally stable DMRs and confirmation of specific DMRs directly correlated with glyphosate resistance will further clarify the role of methylome reprogramming in the evolution of herbicide resistance.

\section{Data accessibility}

All sequencing data and employed scripts can be downloaded from NCBI Sequence Read Archive (SRA) BioProject ID: PRJNA322493.

\section{Acknowledgements}

The authors thank Genomics Research Laboratory (GRL) at Biocomplexity Institute of Virginia Tech for excellent MethylC-seq service.

\section{References}

Akalin A, Kormaksson M, Li S, Garrett-Bakelman FE, Figueroa ME, Melnick A, and Mason CE. 2012. methylKit: a comprehensive R package for the analysis of genome-wide DNA methylation profiles. Genome Biology 13:1-9.

Amrhein N, Deus B, Gehrke P, and Steinrücken HC. 1980. The Site of the Inhibition of the Shikimate Pathway by Glyphosate: II. Interference of glyphosate with chorismate formation in vivo and in vitro. Plant Physiology 66:830-834.

Becker C, Hagmann J, Muller J, Koenig D, Stegle O, Borgwardt K, and Weigel D. 2011. Spontaneous epigenetic variation in the Arabidopsis thaliana methylome. Nature 480:245-249.

Bonny S. 2016. Genetically Modified Herbicide-Tolerant Crops, Weeds, and Herbicides: Overview and Impact. Environmental Management 57:31-48.

Busi R, Neve P, and Powles S. 2013. Evolved polygenic herbicide resistance in Lolium rigidum by low-dose herbicide selection within standing genetic variation. Evolutionary Applications 6:231-242. 
413

414

415

416

417

418

419

420

421

422

423

424

425

426

427

428

429

430

431

432

433

434

435

436

437

438

439

440

441

442

443

444

445

446

447

448

449

450

451

452

453

454

455

456

457

Busi R, and Powles SB. 2009. Evolution of glyphosate resistance in a Lolium rigidum population by glyphosate selection at sublethal doses. Heredity 103:318-325.

Cao X, and Jacobsen SE. 2002a. Locus-specific control of asymmetric and CpNpG methylation by the DRM and CMT3 methyltransferase genes. Proceedings of the National Academy of Sciences 99:16491-16498.

Cao X, and Jacobsen SE. 2002b. Role of the Arabidopsis DRM Methyltransferases in De Novo DNA Methylation and Gene Silencing. Current Biology 12:1138-1144.

Chan SWL, Henderson IR, and Jacobsen SE. 2005. Gardening the genome: DNA methylation in Arabidopsis thaliana. Nat Rev Genet 6:351-360.

Cokus SJ, Feng S, Zhang X, Chen Z, Merriman B, Haudenschild CD, Pradhan S, Nelson SF, Pellegrini M, and Jacobsen SE. 2008. Shotgun bisulphite sequencing of the Arabidopsis genome reveals DNA methylation patterning. Nature 452.

Das M, Reichman JR, Haberer G, Welzl G, Aceituno FF, Mader MT, Watrud LS, Pfleeger TG, Gutiérrez RA, Schäffner AR, and Olszyk DM. 2010. A composite transcriptional signature differentiates responses towards closely related herbicides in Arabidopsis thaliana and Brassica napus. Plant Molecular Biology 72:545-556.

Délye C. 2013. Unravelling the genetic bases of non-target-site-based resistance (NTSR) to herbicides: a major challenge for weed science in the forthcoming decade. Pest Management Science 69:176-187.

Denis M-H, and Delrot S. 1993. Carrier-mediated uptake of glyphosate in broad bean (Vicia faba) via a phosphate transporter. Physiologia Plantarum 87:569-575.

Dowen RH, Pelizzola M, Schmitz RJ, Lister R, Dowen JM, Nery JR, Dixon JE, and Ecker JR. 2012. Widespread dynamic DNA methylation in response to biotic stress. Proceedings of the National Academy of Sciences 109:E2183-E2191.

Duke SO, and Powles SB. 2008. Glyphosate: a once-in-a-century herbicide. Pest Management Science 64:319-325.

Escorial C, Loureiro I, Rodríguez-García E, and Chueca C. 2011. Population Variability in the Response of Ripgut Brome (Bromus diandrus) to Sulfosulfuron and Glyphosate Herbicides. Weed Science 59:107-112.

Espeby LÅ, Fogelfors H, and Milberg P. 2011. Susceptibility variation to new and established herbicides: Examples of inter-population sensitivity of grass weeds. Crop Protection 30:429-435.

Faus I, Zabalza A, Santiago J, Nebauer SG, Royuela M, Serrano R, and Gadea J. 2015. Protein kinase GCN2 mediates responses to glyphosate in Arabidopsis. BMC Plant Biology $15: 14$.

Gaines TA, Zhang W, Wang D, Bukun B, Chisholm ST, Shaner DL, Nissen SJ, Patzoldt WL, Tranel PJ, Culpepper AS, Grey TL, Webster TM, Vencill WK, Sammons RD, Jiang J, Preston C, Leach JE, and Westra P. 2010. Gene amplification confers glyphosate resistance in Amaranthus palmeri. Proceedings of the National Academy of Sciences 107:1029-1034.

González-Torralva F, Rojano-Delgado AM, Luque de Castro MD, Mülleder N, and De Prado R. 2012. Two non-target mechanisms are involved in glyphosate-resistant horseweed (Conyza canadensis L. Cronq.) biotypes. Journal of Plant Physiology 169:1673-1679.

Griffin PT, Niederhuth CE, and Schmitz RJ. 2016. A comparative analysis of 5-azacytidine and zebularine induced DNA demethylation. bioRxiv. 
458

459

460

461

462

463

464

465

466

467

468

469

470

471

472

473

474

475

476

477

478

479

480

481

482

483

484

485

486

487

488

489

490

491

492

493

494

495

496

497

498

499

500

501

502

Hagmann J, Becker C, Müller J, Stegle O, Meyer RC, Wang G, Schneeberger K, Fitz J, Altmann T, Bergelson J, Borgwardt K, and Weigel D. 2015. Century-scale Methylome Stability in a Recently Diverged Arabidopsis thaliana Lineage. PLoS Genet 11:e1004920.

Hansen KD, Langmead B, and Irizarry RA. 2012. BSmooth: from whole genome bisulfite sequencing reads to differentially methylated regions. Genome Biology 13.

Heap I. 2016. The International Survey of Herbicide Resistant Weeds. Online. Internet. Monday, March 21, 2016. Available www.weedscience.org.

Jiang C, Mithani A, Belfield EJ, Mott R, Hurst LD, and Harberd NP. 2014. Environmentally responsive genome-wide accumulation of de novo Arabidopsis thaliana mutations and epimutations. Genome Research 24:1821-1829.

Karan R, DeLeon T, Biradar H, and Subudhi PK. 2012. Salt Stress Induced Variation in DNA Methylation Pattern and Its Influence on Gene Expression in Contrasting Rice Genotypes. PloS One 7:e40203.

Kawakatsu T, Stuart T, Valdes M, Breakfield N, Schmitz RJ, Nery JR, Urich MA, Han X, Lister R, Benfey PN, and Ecker JR. 2016. Unique cell-type-specific patterns of DNA methylation in the root meristem. Nature Plants 2:16058.

Krueger F, and Andrews SR. 2011. Bismark: a flexible aligner and methylation caller for Bisulfite-Seq applications. Bioinformatics 27:1571-1572.

Li S, Garrett-Bakelman FE, Akalin A, Zumbo P, Levine R, To BL, Lewis ID, Brown AL, D'Andrea RJ, Melnick A, and Mason CE. 2013. An optimized algorithm for detecting and annotating regional differential methylation. BMC Bioinformatics 14:1-9.

Li X, Zhu J, Hu F, Ge S, Ye M, Xiang H, Zhang G, Zheng X, Zhang H, Zhang S, Li Q, Luo R, Yu C, Yu J, Sun J, Zou X, Cao X, Xie X, Wang J, and Wang W. 2012. Single-base resolution maps of cultivated and wild rice methylomes and regulatory roles of DNA methylation in plant gene expression. BMC Genomics 13:1-15.

Lin X, Kaul S, Rounsley S, Shea TP, Benito M-I, Town CD, Fujii CY, Mason T, Bowman CL, Barnstead M, Feldblyum TV, Buell CR, Ketchum KA, Lee J, Ronning CM, Koo HL, Moffat KS, Cronin LA, Shen M, Pai G, Van Aken S, Umayam L, Tallon LJ, Gill JE, Adams MD, Carrera AJ, Creasy TH, Goodman HM, Somerville CR, Copenhaver GP, Preuss D, Nierman WC, White O, Eisen JA, Salzberg SL, Fraser CM, and Venter JC. 1999. Sequence and analysis of chromosome 2 of the plant Arabidopsis thaliana. Nature 402:761-768.

Lu YC, Feng SJ, Zhang JJ, Luo F, Zhang S, and Yang H. 2016. Genome-wide identification of DNA methylation provides insights into the association of gene expression in rice exposed to pesticide atrazine. Scientific Reports 6:18985.

Matzke MA, and Mosher RA. 2014. RNA-directed DNA methylation: an epigenetic pathway of increasing complexity. Nat Rev Genet 15:394-408.

Mirouze M, and Vitte C. 2014. Transposable elements, a treasure trove to decipher epigenetic variation: insights from Arabidopsis and crop epigenomes. Journal of Experimental Botany 65:2801-2812.

Nardemir G, Agar G, Arslan E, and Aygun Erturk F. 2015. Determination of genetic and epigenetic effects of glyphosate on Triticum aestivum with RAPD and CRED-RA techniques. Theoretical and Experimental Plant Physiology 27:131-139.

Niederhuth CE, Bewick AJ, Ji L, Alabady MS, Kim KD, Li Q, Rohr NA, Rambani A, Burke JM, Udall JA, Egesi C, Schmutz J, Grimwood J, Jackson SA, Springer NM, and Schmitz RJ. 
2016. Widespread natural variation of DNA methylation within angiosperms. Genome Biology 17:194.

Okada M, Hanson BD, Hembree KJ, Peng Y, Shrestha A, Stewart CN, Wright SD, and Jasieniuk M. 2013. Evolution and spread of glyphosate resistance in Conyza canadensis in California. Evolutionary Applications 6:761-777.

Quinlan AR, and Hall IM. 2010. BEDTools: a flexible suite of utilities for comparing genomic features. Bioinformatics 26:841-842.

Sammons RD, and Gaines TA. 2014. Glyphosate resistance: state of knowledge. Pest Management Science 70:1367-1377.

Schmitz RJ, Schultz MD, Lewsey MG, O’Malley RC, Urich MA, Libiger O, Schork NJ, and Ecker JR. 2011. Transgenerational Epigenetic Instability Is a Source of Novel Methylation Variants. Science 334:369-373.

Secco D, Wang C, Shou H, Schultz MD, Chiarenza S, Nussaume L, Ecker JR, Whelan J, and Lister R. 2015. Stress induced gene expression drives transient DNA methylation changes at adjacent repetitive elements. eLife 4.

Stroud H, Greenberg Maxim VC, Feng S, Bernatavichute Yana V, and Jacobsen Steven E. 2013. Comprehensive Analysis of Silencing Mutants Reveals Complex Regulation of the Arabidopsis Methylome. Cell 152:352-364.

Tzin V, and Galili G. 2010. The Biosynthetic Pathways for Shikimate and Aromatic Amino Acids in Arabidopsis thaliana. The Arabidopsis Book:e132.

Verhoeven KJF, Jansen JJ, van Dijk PJ, and Biere A. 2010. Stress-induced DNA methylation changes and their heritability in asexual dandelions. New Phytologist 185:1108-1118.

Wibowo A, Becker C, Marconi G, Durr J, Price J, Hagmann J, Papareddy R, Putra H, Kageyama J, Becker J, Weigel D, and Gutierrez-Marcos J. 2016. Hyperosmotic stress memory in Arabidopsis is mediated by distinct epigenetically labile sites in the genome and is restricted in the male germline by DNA glycosylase activity. eLife 5:e13546.

Yong-Villalobos L, González-Morales SI, Wrobel K, Gutiérrez-Alanis D, Cervantes-Peréz SA, Hayano-Kanashiro C, Oropeza-Aburto A, Cruz-Ramírez A, Martínez O, and HerreraEstrella L. 2015. Methylome analysis reveals an important role for epigenetic changes in the regulation of the Arabidopsis response to phosphate starvation. Proceedings of the National Academy of Sciences 112:E7293-E7302.

$\mathrm{Yu}$ X, and Sun S. 2016. Comparing five statistical methods of differential methylation identification using bisulfite sequencing data. Statistical Applications in Genetics and Molecular Biology 15:173-191.

Zemach A, Kim MY, Hsieh P-H, Coleman-Derr D, Eshed-Williams L, Thao K, Harmer Stacey L, and Zilberman D. 2013. The Arabidopsis Nucleosome Remodeler DDM1 Allows DNA Methyltransferases to Access H1-Containing Heterochromatin. Cell 153:193-205.

Żywicki M, Gracz J, Karłowski W, Twardowski T, and Tyczewska A. 2015. Expression of miRNAs involved in phosphate homeostasis and senescence is altered in glyphosatetreated maize. Acta Physiologiae Plantarum 37:1-10.

\section{Figure Legends}

Figure 1. Overview of experimental pipeline. A) Effect of 0, 5, 10, and $15 \%$ of a $0.9 \mathrm{~kg}$ ae ha-1 field rate of glyphosate on $A$. thaliana floral shoot development. B) Schematic of glyphosate treatment and tissue for harvesting gDNA. C) Pipeline for analyzing methylC-seq data to identify 
548 differentially methylated cytosines (DmCs) and differentially methylated regions (DMRs). D)

549 Example of DMRs identified by bsseq. Blue lines represent methylation levels across the

550 window of individual replicates of control plants and red lines represent methylation levels

551 across the window of individual $5 \%$ or $10 \%$ glyphosate-treated plants.

552 Figure 2. Global pattern of methylated cytosines ( $\mathrm{mCs}$ ) and differentially methylated cytosines

553 (DmCs) following methylkit pipeline. A) Relative abundance of $\mathrm{mCs}$ in the three sequence

554 contexts (CG, $\mathrm{CHG}, \mathrm{CHH}$ ) following 0,5 or $10 \%$ of a $0.9 \mathrm{~kg}$ ae ha-1 glyphosate treatment to 4-

555 week old $A$. thaliana rosettes. $\mathrm{n}=4$ plants for each treatment. B-C) Relative abundance of hyper-

556 and hypo-methylated DmCs in the 5\% and 10\% glyphosate-treated samples compared to the $0 \%$

557 controls across all chromosomes in the CG (B), CHH (C), and CHG (D) sequence contexts. See

558 Dataset S1 for list of all DmCs.

559 Figure 3. Identification and dose-dependency of DMRs. A) overlap of DMRs between the 5\%

560 and $10 \%$ glyphosate treatment groups by sequence context. B) Number of $5 \%$ and $10 \%$

561 overlapping DMRs that exhibit glyphosate dose-independent (panel 1), positive dose-dependent

562 (panel 2), or inverse dose-dependent (panel 3) methylation responses based on non-parametric

563 curve fitting and statistical test for significant difference between the doses at $p<0.05$. All DMRs

564 are described in Dataset S3. The list of dosage classification for all overlapping DMRs is

565 available in Dataset S4. Glyphosate percentages based on a $0.9 \mathrm{~kg}$ ae ha-1 rate.

566 Figure 4. Location of DMRs in the $A$. thaliana genome and comparison to DMRs induced by

567 other stresses. A) Prevalence of DMRs by annotation context in the $A$. thaliana genome. See

568 Dataset S3 for annotated list of all DMRs. B) and C) Gene ontology (GO) terms for biological

569 process $(B)$ and molecular function $(C)$ enriched $(\mathrm{p}<0.05)$ in DMR associated genes. $\mathrm{Rf}_{\mathrm{i}} / \mathrm{Rf}_{\mathrm{r}}$

570 represents the ratio of the relative frequency of GO terms in the input (glyphosate DMRs) to the

571 reference (TAIR10 Arabidopsis genome) datasets. * indicates $\mathrm{p}<0.01$. Redundant GO terms

572 excluded from the figure. See Dataset $\mathrm{S} 6$ for full list of GO terms with $\mathrm{Rf}_{\mathrm{i}} / \mathrm{Rf}_{\mathrm{r}}>1.5$. D)

573 Comparison of DMR-associated genes identified following glyphosate stress compared to DMR-

574 associated genes previously identified as induced by biotic stress mimic (Dowen et al. 2012) or

575 phosphate starvation (Yong-Villalobos et al. 2015). Dataset S8 lists all DMR-associated genes

576 categorized by overlap (or lack thereof) among the three analyzed stresses. 


\section{Figure 1}

Figure 1. Overview of experimental pipeline.

A) Effect of $0,5,10$, and $15 \%$ of a $0.9 \mathrm{~kg}$ ae ha- ${ }^{-1}$ field rate of glyphosate on $A$. thaliana floral shoot development. B) Schematic of glyphosate treatment and tissue for harvesting gDNA. C) Pipeline for analyzing methylC-seq data to identify differentially methylated cytosines (DmCs) and differentially methylated regions (DMRs). D) Example of DMRs identified by bsseq. Blue lines represent methylation levels across the window of individual replicates of control plants and red lines represent methylation levels across the window of individual $5 \%$ or $10 \%$ glyphosate-treated plants.

A

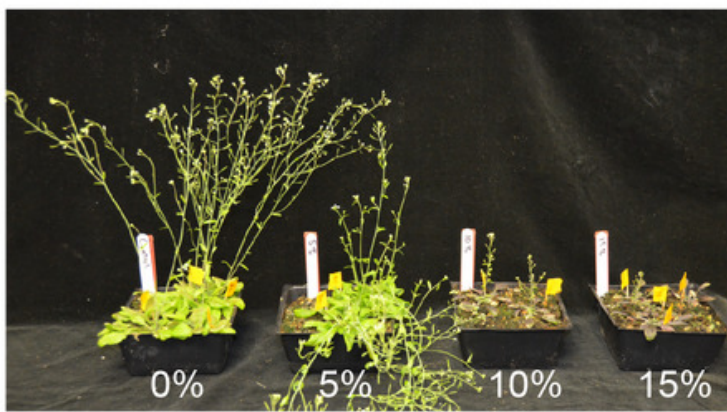

C

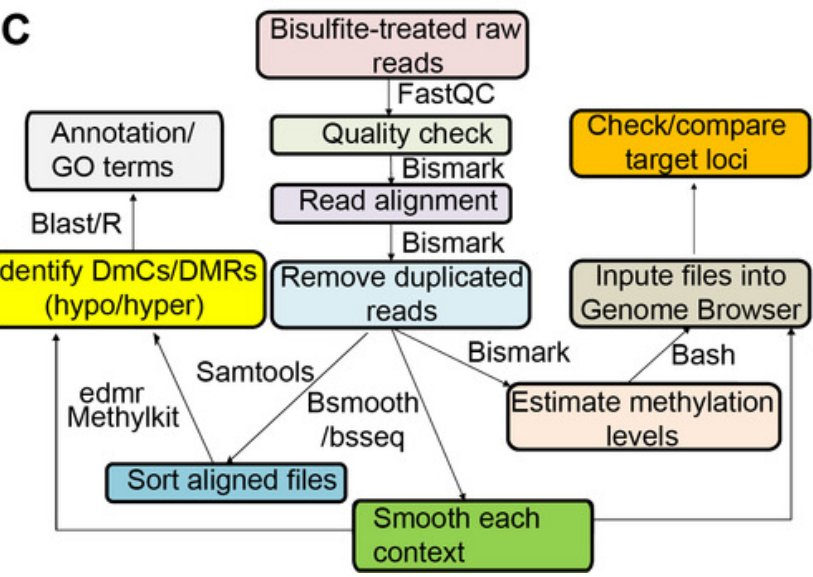

B individual plant

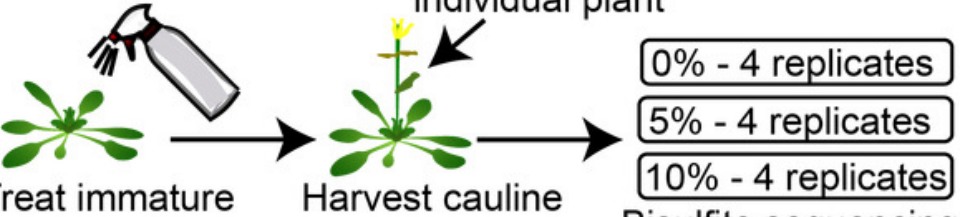

Treat immature A. thaliana with glyphosate leaves at silique Bisulfite sequencing development

( 2 weeks for $0 \%$ and $5 \%$ plants, 8 weeks for $10 \%$ plants)

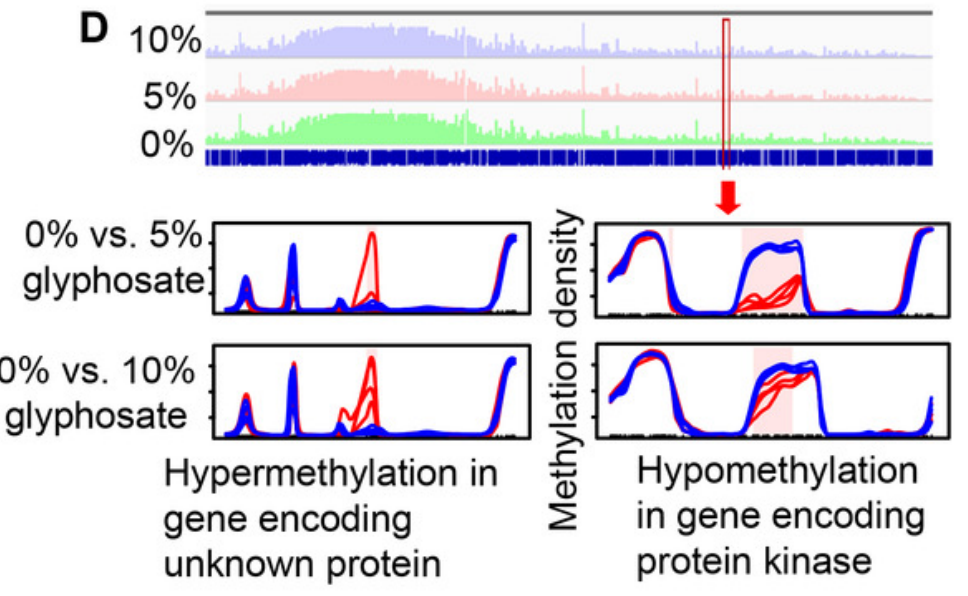




\section{Figure 2}

Figure 2. Global pattern of methylated cytosines (mCs) and differentially methylated cytosines (DmCs) following methylkit pipeline.

A) Relative abundance of $\mathrm{mCs}$ in the three sequence contexts ( $\mathrm{CG}, \mathrm{CHG}, \mathrm{CHH}$ ) following 0,5 or $10 \%$ of a $0.9 \mathrm{~kg}$ ae ha- ${ }^{-1}$ glyphosate treatment to 4 -week old $A$. thaliana rosettes. $n=4$ plants for each treatment. B-C) Relative abundance of hyper- and hypo-methylated DmCs in the $5 \%$ and $10 \%$ glyphosate-treated samples compared to the $0 \%$ controls across all chromosomes in the CG (B), CHH (C), and CHG (D) sequence contexts. See Dataset S1 for list of all DmCs. 
A

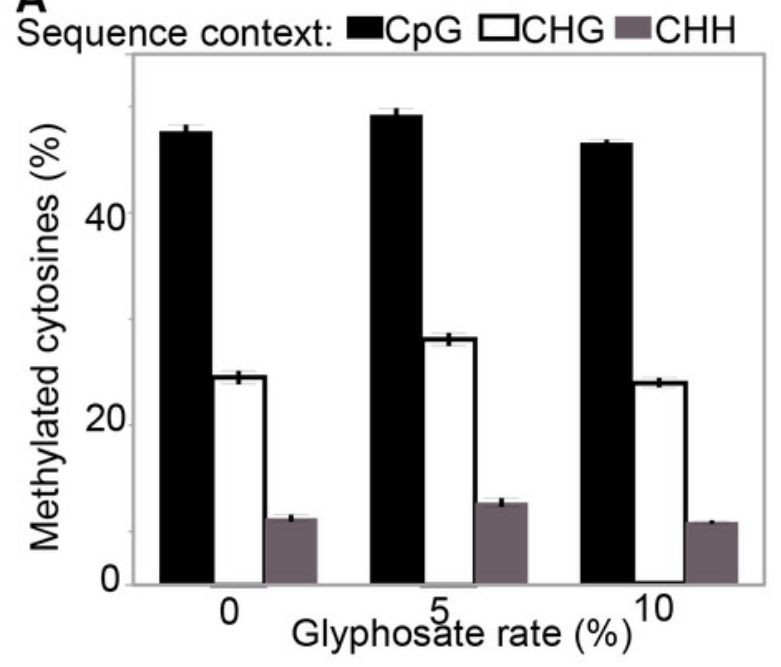

Hypermethylation

Hypomethylation

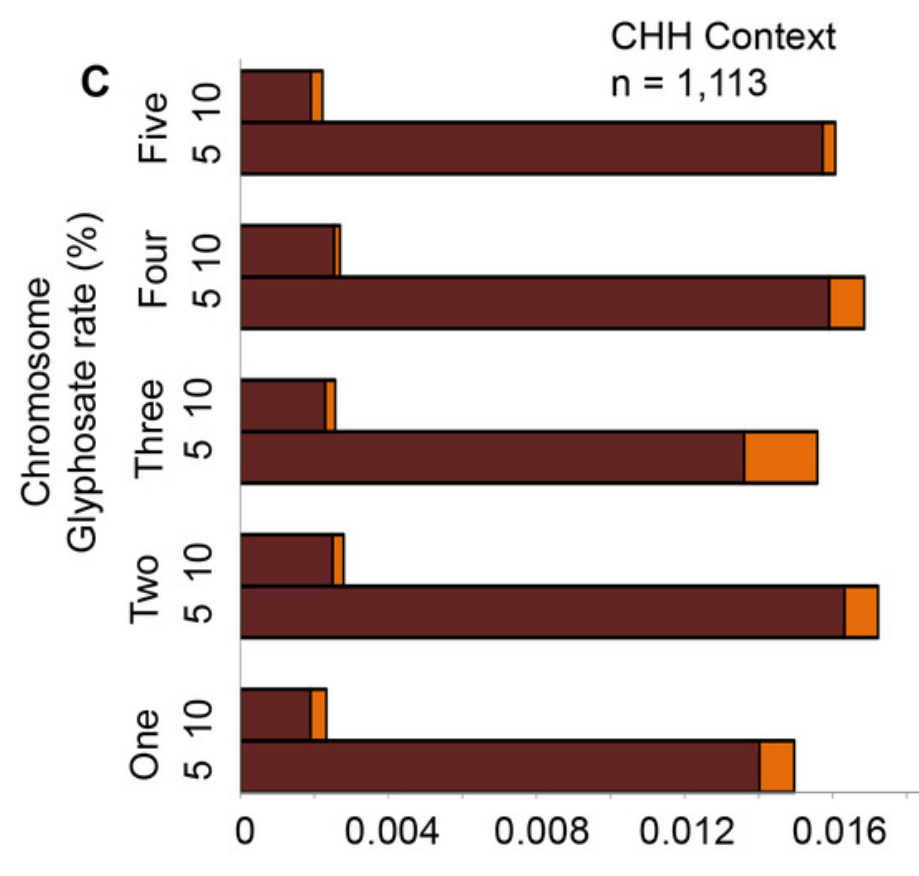

Differentially methylated cytosines (\% of all methylated cytosines)
B CG Context
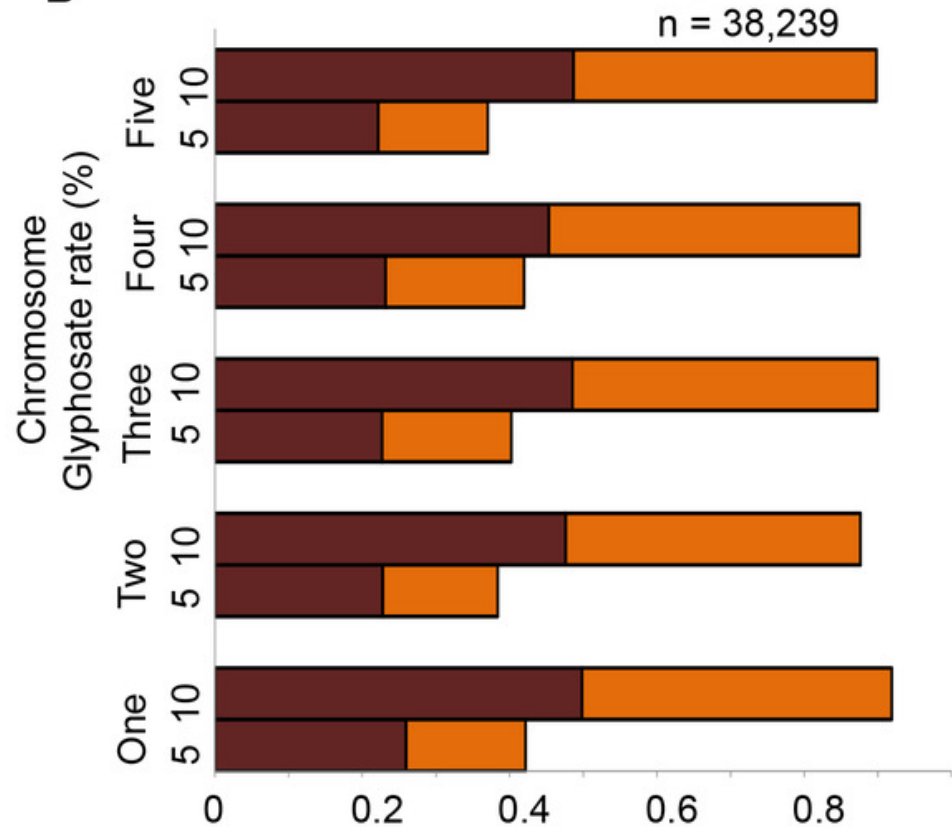

Differentially methylated cytosines ( $\%$ of all methylated cytosines)

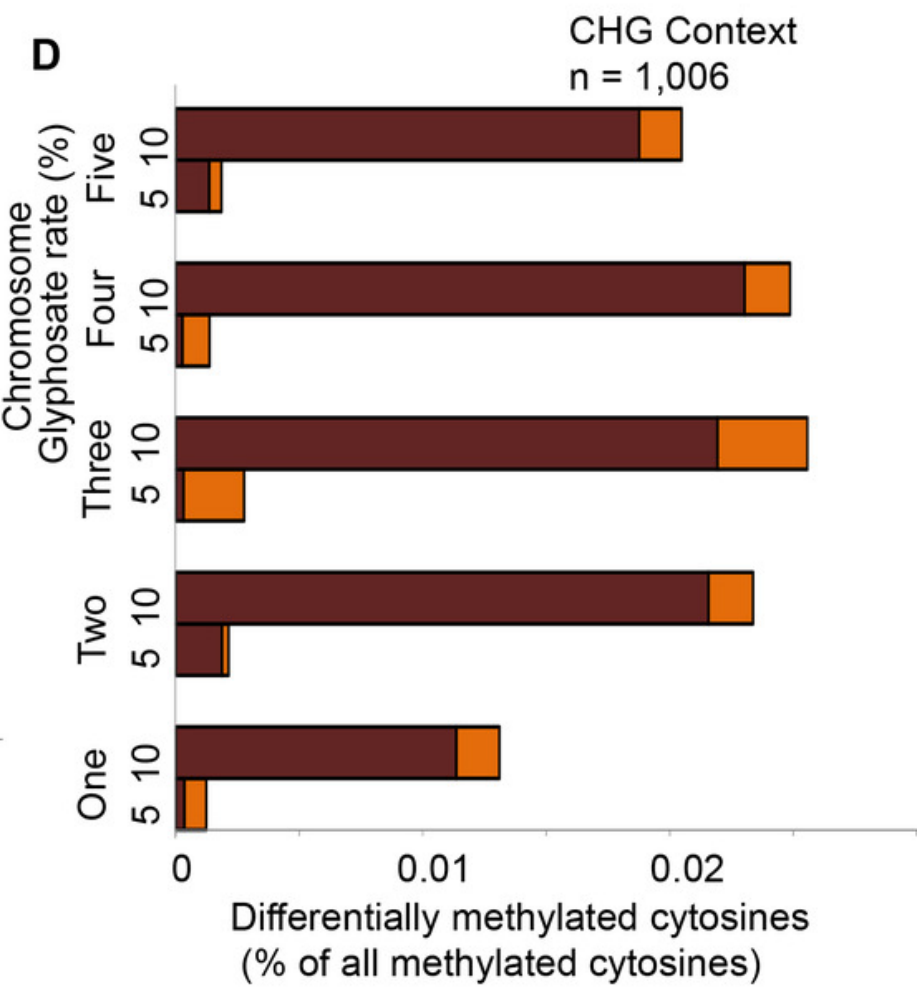




\section{Figure 3}

Figure 3. Identification and dose-dependency of DMRs.

A) overlap of DMRs between the $5 \%$ and $10 \%$ glyphosate treatment groups by sequence context. B) Number of $5 \%$ and $10 \%$ overlapping DMRs that exhibit glyphosate doseindependent (panel 1), positive dose-dependent (panel 2), or inverse dose-dependent (panel 3) methylation responses based on non-parametric curve fitting and statistical test for significant difference between the doses at $p<0.05$. All DMRs are described in Dataset S3. The list of dosage classification for all overlapping DMRs is available in Dataset S4. Glyphosate percentages based on a $0.9 \mathrm{~kg}$ ae ha-1 ${ }^{-1}$ rate. 
A
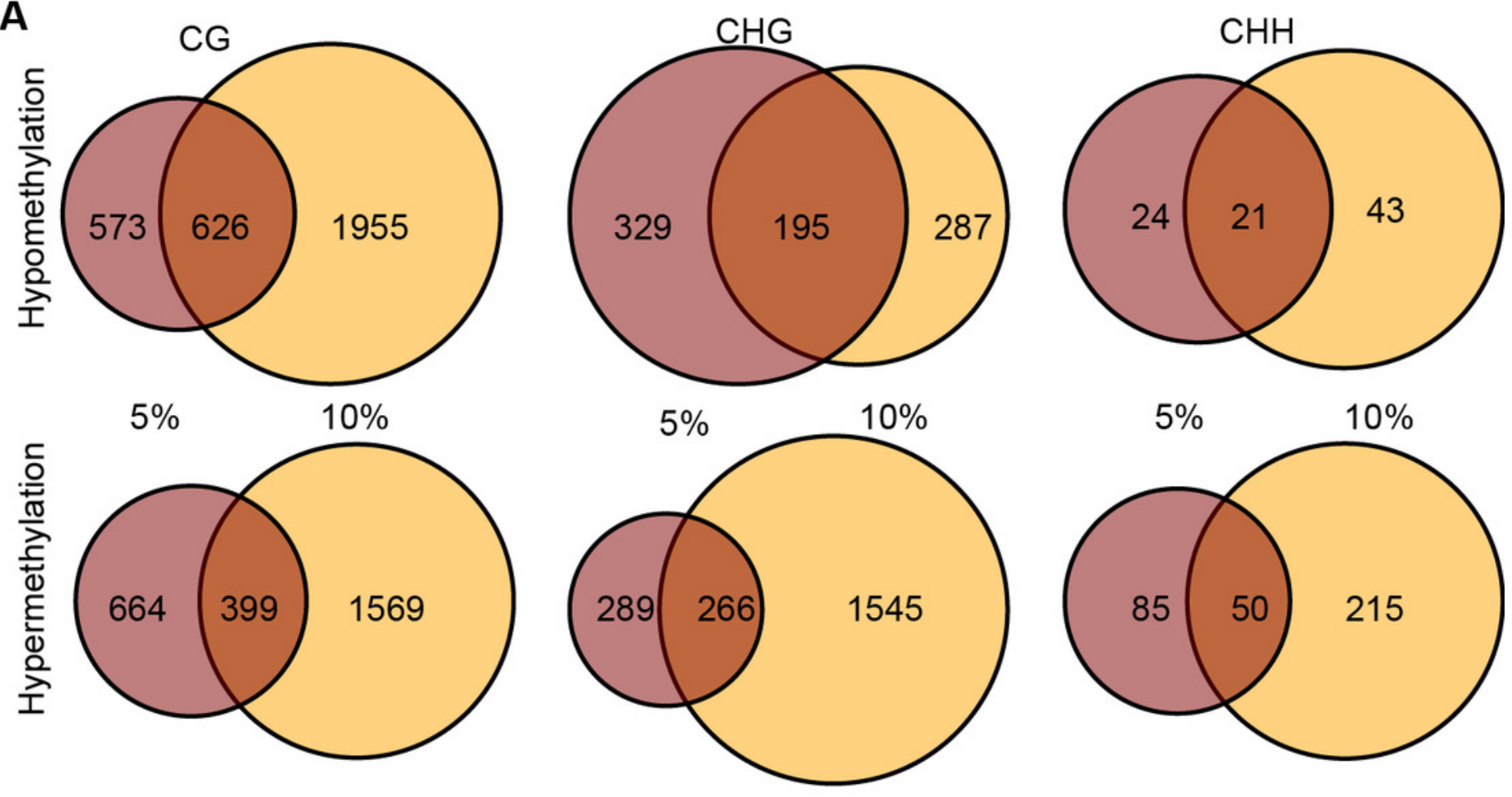

B
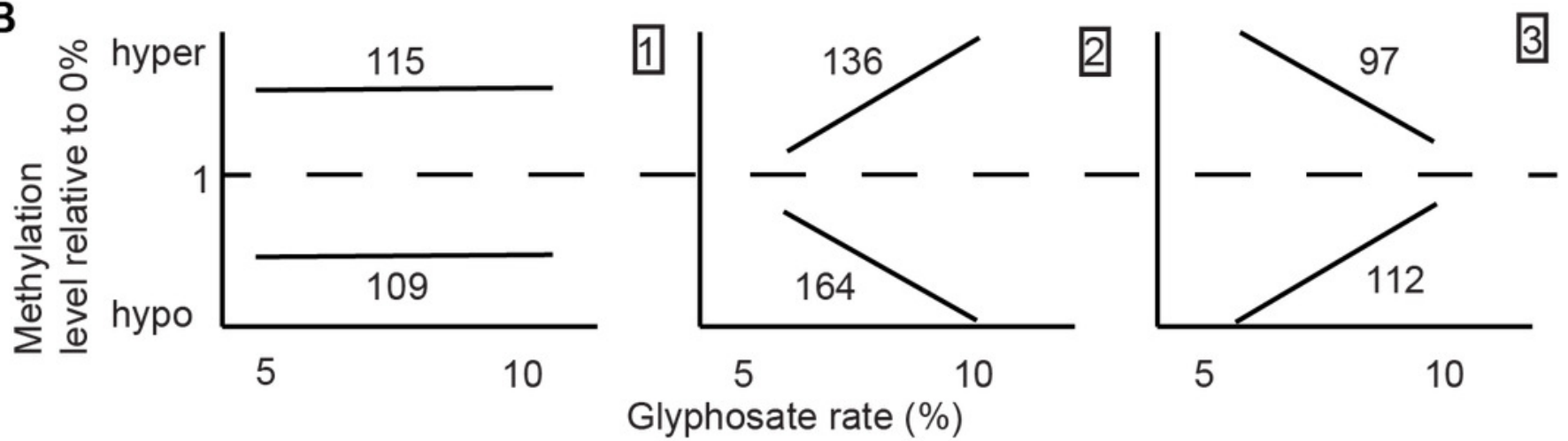


\section{Figure 4}

Figure 4. Location of DMRs in the $A$. thaliana genome and comparison to DMRs induced by other stresses.

A) Prevalence of DMRs by annotation context in the A. thaliana genome. See Dataset S3 for annotated list of all DMRs. B) and C) Gene ontology (GO) terms for biological process (B) and molecular function $(C)$ enriched $(p<0.05)$ in $D M R$ associated genes. $R f_{i} / R f_{r}$ represents the ratio of the relative frequency of GO terms in the input (glyphosate DMRs) to the reference (TAIR10 Arabidopsis genome) datasets. * indicates $p<0.01$. Redundant GO terms excluded from the figure. See Dataset $\mathrm{S} 6$ for full list of $\mathrm{GO}$ terms with $\mathrm{Rf}_{\mathrm{i}} / \mathrm{Rf}_{\mathrm{r}}>1.5$. D) Comparison of DMR-associated genes identified following glyphosate stress compared to DMR-associated genes previously identified as induced by biotic stress mimic (Dowen et al. 2012) or phosphate starvation (Yong-Villalobos et al. 2015) . Dataset S8 lists all DMR-associated genes categorized by overlap (or lack thereof) among the three analyzed stresses.
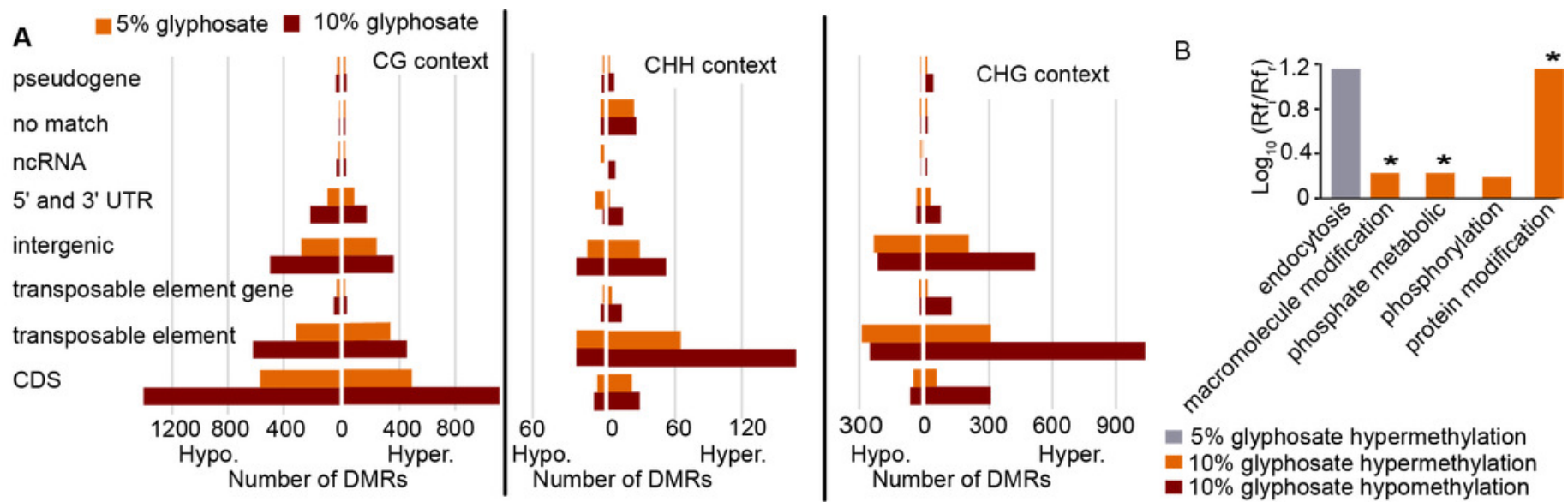

C
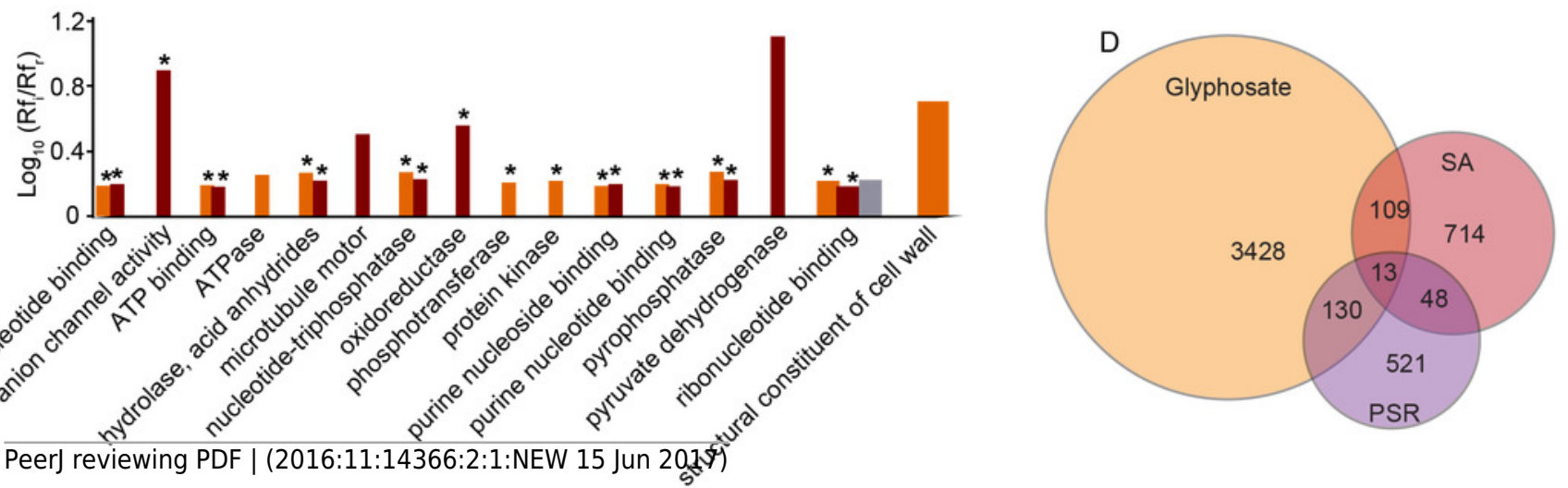